[1] P. Erdös and I. S. Gál, On the law of the iterated logatithm, Proo. Arnsterdaun 58 (1955), pp. 65-84.

[2] L. Gál and. S. Gál, The discrepanoy of the sequence $\left\{2^{n} x\right\}$, ilid. (17 (1964), pp. 129-143.

[3] L. Kuipers and H. Nioderreiter, Uniform aistribntion of sequences, John Wiley \& Sons, New York-London 1974.

[4] H. G. Meijer, The discrepanoy of a g-adire sequande, I'roc. Amsterdiun 7l (I968), pp. 54-60.

[5] W. PLilipp, Hixing sequences of rankthm voriables and prothabilistic namber theory, Momoirs AMS 114, Providenec, R. T., 1.971.

[0] - Limit theorems for lacunary series and whiforn distrinution mod 1, Aeta Aritb. 26 (1975), pp. 241-251.

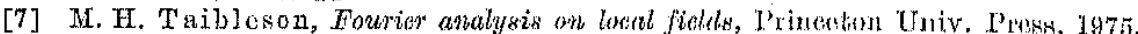

DEPARTMENT OF MLAITEMMATICOS

THEE UNIVERSTYY OF ITEXAS

Austin, Texas 78712

\section{Class number formulas for quaternary quadratic forms}

by

\author{
PaUd PONOMarit* (Columbus, Ohio)
}

Introduction. This penex may be regarded as a secuncl to [4]. Unlcss otherwise indicated, the motation and terminology aro taken from [4], especially $\$ 1, \$ 3$ and $\$ 5$.

We recapitulate some of tho results on class numbers derived in [3], [4]. Let $V$ be a definite quadratic space of dimension four over the field of rational numbers $Q$. Let $\mathfrak{I}$ be an idealcomplex of maximal latices on $V$ (af. [4], §3). Let $\Delta$ denote the reduced discriminant of $\Im$ and $H$ the number of proper similitude classes in $\mathfrak{J}$. In the case where $V$ has square discriminant $\mathfrak{\Im}$ is uniquely determincd, and an explicit formula for $H$ was given in [3] (Theorem, p. 297).

If the discriminant $D(Y)$ of $V$ is not a square, we put $K=Q(\sqrt{D(V)})$, and denoto the discriminant of $K$ by $\Delta_{K}$. It was shown in [4] (Prop. 7 ) that

$$
\Delta=\Delta_{K}\left(p_{1} \ldots p_{e}\right)^{2}\left(q_{1} \ldots q_{f}\right)^{2}
$$

where $q_{1}, \ldots, q_{f}$ are the anisotropic finite primes of $V ; q_{1}, \ldots, q_{f}$ split in $K$, and $p_{1}, \ldots, p_{e}$ are distinct rational primes which remain prime in $K$. In $\S 6$ of $[4]$ explicit tormulas were obtained for $H$ (Theorems 1, 2) under the following conditions:

(i) $f=0$,

(ii) The fundamental unit of $K$ has norm -1.

In this paper wo obtain such formulas for $H$ without making either of these restrictions. As a restitt, we completely solve the problem of determining the proper class number of an arbitrary idealcomplex of maximal quateroary lattices (ar. [4], Prop. 11, for the indefinito caso). As a special case of these lormulas wo obtain, in the classical language, a formula for the mumber of proper alnsses of positivo definite integral quaternary forms of discriminant $\Delta_{K}$.

$\mathrm{By}$ coling, lattices of $V$. When $D(V)$ is s nonsquare, there is a unique quaternion

* Partially supported by NST grant MCS 76-08746 A01. 


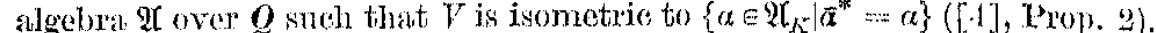
Then $p_{1}, \ldots, p_{\varepsilon} ; q_{1}, \ldots, q_{f}$ may be characterized as the set of all rational primes which ramify in 2 but not in $K$ ([4], Prop. $6(\mathrm{~b}))$. We set $\delta_{1}=p_{1} \ldots$ $\ldots p_{e}, \delta_{2}=q_{1} \ldots q_{f}, \delta=\delta_{1} \delta_{2}$. Then $H$ may be identified with a $\mathrm{g} \cdot \mathrm{n}-$ eralized type number $t_{s}(\Omega)$, where $\Omega$ is an lajchler ordex of $\mathfrak{A}_{K}$ of level $\delta$ $\left([4]\right.$, p. 22). If $\delta_{2}=1$, then $t_{8}(\Omega)=t(\Omega)$, the nsual type number of $\Omega$ Thus the formulas we obtain are also formulas for the typo numbers of certain Eichler orders associated to $\mathfrak{A}_{K}$. Onder tho turther restriction that $\delta=1$, Vigneras [5] stated a formmla for $t(\Omega)$ which is incorrect, in general. The correct formula is the special caso $e=f \ldots 0$ of our tormulas in Section 5 .

1. Tet $a m a^{*}$ be the cmonical involution of $\mathfrak{A f}_{K}$ and $N: \mathfrak{A}_{K^{\prime} \rightarrow K} K_{\text {the }}$ rednced norm. The conjugation map of $K$ extends to a $Q$-automorphism. $a \mapsto \rightarrow \bar{a}$ of $\mathfrak{A}_{\mathcal{K}}$ having $\mathfrak{O}$ as its fixed ring. These mappings extend in the obvious manner to the completions of $\mathfrak{A}_{K}$ and to its idele group $J_{\mathfrak{O}_{K}}$. Let $J_{Q}^{1}, J_{K}^{1}, J_{\mathfrak{I}_{K}}^{1}$ denote the norm 1 idlele groups of $Q, K, \mathfrak{2}_{K}$, respectively. Let $\Omega$ be an Eichler order of $\mathfrak{A}_{K}$ of level $\delta$ which is symmetric, in tho senso that $\bar{\Omega}=\Omega$. The symmetric normalizer $\mathfrak{N}_{s}^{1}(\tilde{\Omega})$ is defined to bo the group of all $\nu \in J_{\mathfrak{V}_{K}}^{1}$ such that $v \tilde{\Omega} \tilde{v}^{*}=n \tilde{\Omega}$ for some $n \in J_{Q}^{1}$ (ct. [4], p. 21). The usual normalized $\mathfrak{R}^{1}(\tilde{\Omega})$ is defined to bo the group of: all $\eta^{\prime} \in J_{\mathfrak{U}_{K}}^{1}$ such that $\nu \hat{\Omega} \nu^{-1}=\tilde{\Omega}$. One etisily sees that $\mathfrak{R}_{g}^{1}(\tilde{\Omega})$ is in normal knl)group of $\mathfrak{R}^{\mathfrak{l}}(\tilde{\Omega})$ and $\mathfrak{R}^{\mathrm{l}}(\tilde{\Omega}) / \mathfrak{M}_{8}^{\mathrm{l}}(\tilde{\Omega})$ is an elementary abelinn 2 -group of onder $2^{f}([4] ;$ p. 22). Set

$$
G=\mathfrak{Y}_{K}^{\times} / K^{\times}, \quad G_{s t}^{1}=J_{\mathfrak{V}_{K}}^{1} / J_{K}^{1}, \quad G_{s}^{1}(\tilde{\Omega})=\mathfrak{N}_{s}^{1}(\tilde{\Omega}) / J_{K^{\circ}}^{1} .
$$

The generalized type number $t_{s}(\Omega)$ is delined by

$$
t_{s}(\Omega)=\operatorname{card}\left(G \backslash G_{A}^{1} / G_{s}^{1}(\tilde{\Omega})\right) .
$$

It was shown in $\$ 4$ of [4] that $H=t_{8}(\Omega)$ (Prop. 9).

For every rational prime $p$ let $K_{p}=K \otimes_{Q} \boldsymbol{Q}_{p}, \mathfrak{A}_{K_{p}}=\mathfrak{A} \otimes_{Q} K_{p}$. If $K_{p}$ is a direct sum of two fields, thon $\boldsymbol{Q}_{p}$ is identified with the diagonal of $K_{p}$. We denote the unit group of $K_{p}$ loy $U_{K_{p}}$. From the local description of the symmetrie normalizer ([4], p. 22) it is readily seen that $\mathfrak{R}^{1}(\tilde{\Omega})$ is the set of all $y=\left(y_{p}\right) \in \mathfrak{R}^{1}(\tilde{\Omega})$ such that

$$
N\left(v_{p}\right) \in Q_{j}^{\times}\left(K_{, p}^{\times}\right)^{2} U_{K_{p}} \text { for all } p \mid \delta_{2} .
$$

Whe latter norm description can be used to define $\mathfrak{N}_{\mathrm{s}}^{1}(\tilde{\Omega}), t_{\mathrm{g}}(\Omega)$ for any Eichlex order of level $\delta$, symmetric or not. Since all Wichler orders of level $\delta$ are locally conjugate, $t_{s}(\Omega)$ is independent of the choice of $\Omega$. We denote this common valuo by $t_{s}$, and the usual typo number of Wichler orders of level $\delta$ by $t$.
We fix $\Omega$ and let $L_{2}\left(G \backslash G_{A}^{\mathrm{l}} / G_{s}^{\mathrm{I}}(\tilde{\Omega})\right)$ denote the space of all complexvalued functions on $G_{A}^{1}$ which stre invariant under left multiplieation by $G$ and right multiplication by $G_{s}^{x}(\tilde{\Omega})$. Let $F_{\tilde{\Omega}}$ bo the characteristic function of $G_{s}^{1}(\tilde{\Omega})$. If we normalize the Faar mewsure on $G_{A}^{1}$ so that $G_{s}^{1}(\tilde{\Omega})$ has moasure 1 , then convolution with respect to $F_{\tilde{\Omega}}$ gives the identity operator on $L_{s}\left(G \backslash Q_{A}^{1} \mid Q_{s}^{1}(\tilde{\Omega})\right)$. Applying the Selberg trace formula as in $\$ 8$ of [4], we obltain

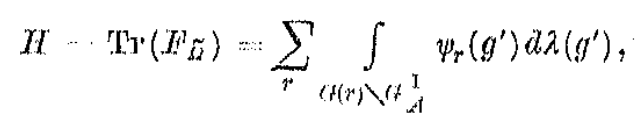

where $r$ runs over a complete set of representatives for the conjugacy dasses of $Q, G^{*}(r)$ is the centralizer of $r$ in $q$, and

$$
\psi_{r}\left(g^{\prime}\right)=F_{\tilde{\Omega}}\left(g^{-1} r g\right), \quad g \in Q_{A !}^{1} .
$$

A representative 9 makes a non-zero contribution to the trace sum (2) if and only if $g^{-1} r g \in G_{s}^{1}(\tilde{\Omega})$ for some $g \in G_{A}^{1}$. Let $\alpha \in \mathfrak{F}_{K}$ represent $r$, and $\gamma \in J_{\mathbb{V}_{K}}^{1}$ represent $g$. The 11

$g^{-1} r g \in G_{s}^{1}(\tilde{\Omega}) \Leftrightarrow \gamma^{-1} \alpha \gamma \in \mathfrak{R}_{s}^{y}(\tilde{\Omega}) \Leftrightarrow \gamma^{-1} a \gamma \in \mathfrak{R}^{1}(\tilde{\Omega})$ and

$N\left(\gamma_{p}^{-1} \alpha_{p} \gamma_{p}\right) \in Q_{p}^{\times}\left(K_{p}^{\times}\right)^{2} U_{K_{p}}$ for all $p \mid \delta_{2} \Leftrightarrow \alpha \in \mathfrak{N}^{2}\left(\gamma \tilde{\Omega} \gamma^{-1}\right)$ and $N\left(\alpha_{p}\right) \in Q_{p}^{\times}\left(K_{p}^{\times}\right)^{2} U_{K_{p}}$ for all $p \mid \delta_{2} \Leftrightarrow \alpha \in \mathfrak{N}\left(\gamma \Omega \gamma^{-1}\right)$ and the principal $(N(\alpha))=n \mathfrak{i}^{2}$, where $n$ is a rational integer dividing $\delta$, and $\mathfrak{t}$ is a tractional ideal of: $K$.

2. Wo proceed now to determine all $\alpha$ which give non-zero contributions to the trace sum (2). Our approach is a modified form of the argument in $\S 7$ of [1]. If $\xi_{1}, \xi_{2}$ are algebrais numbers, then $\xi_{1} \simeq \xi_{2}$ will mean that, for some $x \in K^{\prime \prime}, x \xi_{1}$ and $\xi_{2}$ have the same minimal polynomial over $K$. If $\alpha, \beta \in \mathfrak{H}_{K}$, the condition $\alpha \simeq \beta$ is equivalent to the classes of $\alpha, \beta$ being conjugate in $\theta$.

Suppose $\alpha \neq 1$, which is to say $\alpha \notin K$. Wo may assume $\alpha$ is integral over $D$, the ring of integers of $K$. Then $(N(\alpha))=n i^{3}$, where $t$ is an jntegral ideal of $D$ and to is a rational integex dividing $\delta$. From $\mathfrak{i}^{2}=\left(n^{-1} N(\alpha)\right)$ it follows that $i$ belongs to an ambignous ideal class of $K$. Lot $D$ denote the square-fres kemel of $A_{K}$. Then one of the following mont hold ([1], p. 190, Exx. 8-10)

(a) $\mathfrak{i}$ is equivalent to an ambiguons ideal,

(b) $D=a^{2}+b^{2}$, where $a, b$ are integers; every unit of $D$ has norm 1 , and $i=x a$, where $x \in K$ and $a^{2}=(b+\sqrt{D})$.

If (b) holds, then $(N(\alpha))=\left(n x^{2}(b+\sqrt{D})\right)$. Taking the norm of both sides, wo obtain

$$
n_{K / Q}(N(\alpha))=n^{2}\left(n_{K / Q}(x)\right)^{2}\left(b^{2}-D\right),
$$

7 - Acta Arithmetica $\mathrm{xxxrx} \mathrm{z}_{1} 1$ 
since all units have norm 1. This implies

$$
n_{K / \Omega}(N(\alpha))=n^{2}\left(n_{K / Q}(x)\right)^{2}\left(-a^{2}\right),
$$

contradicting the fact that $N(a)$ is totally positive. Since (a) is the only possibility, the reasoning of $\$ 7$ in [4] is valid here, and we dedueo that $(N(a))=n \mathrm{j}^{2}$, where $\mathrm{j}$ is ambiguous. Thus $N(a) \ldots m u$, where $m$ is an squure free rational integer dividing $\delta A_{K}$, and $u$ is at totally positive unit of $D$.

The minimal polynomial of $a$ over $K$ must then be of the form $X^{2}+$ $+b X+m u$, where $b \in \mathcal{D}$. Arguing waxtly as in $\$ 7$ of: [4], we have $\alpha^{*}=\omega^{-1} \alpha$, where $\omega$ is a primitive $n$th root of 1 for one of the following values of $n: 2,3,4,5,6,8,10,12$. If $y_{t}=2$, then $b \ldots 0$ and wo have $\alpha \simeq \sqrt{-m u}$.

If $n>2$, then $K(a)=K(\omega)$ and $a^{2}=m u \omega$. It sulfieces to considar the equation $a^{2}= \pm m u \omega$ in $K(\omega)$ for $n=3,4,5,8,12$. Wo note thist $D$ must be 5 if $n=5, D=2$ if $n=8$, and $D=3$ if $n=12$. Put $a=x+$ $+y \omega, x, y \in K$. Wach possible $\omega$ satisfies a minimal polynomial over $K$ having constant term 1. This inflies $a^{2}=x^{2}-y^{2}+z \omega, z \in K$. Fonce $x^{2}-y^{2}-1-z \omega= \pm m u \omega$, from which it follows that $x^{2}=y^{2}, x= \pm y$. If $x=0$, then $\alpha \simeq \omega$. If $x \neq 0$, then $a \simeq 1 \pm \omega$. Wo consider the various possibilities:

If $n=3$, then $\alpha \simeq \zeta$, a primitive cube root of 1 , or $a \simeq 1-\zeta \simeq \zeta \sqrt{-3}$

If $n=4$, then $\alpha \simeq \sqrt{-1}$ or $a \simeq 1+\sqrt{-1}$.

If $n=5$, let $\eta$ denote a primitive 5 -th root of 1 . Wo olserve that $1+\eta \simeq \eta$. Furthermore, $N(1-\eta)=\sqrt{5} v$ for some unit $v$ of $D$, which means $1-\eta$ cannot normalize an Eichler order of level $\delta$. Tuet $\eta^{\prime}$ denote another primitive 5 -thl root which is not conjugate to $\eta$ over $Q(\sqrt{5})$. Then $\alpha \simeq \eta$ or: $\alpha \simeq \eta^{\prime}$.

If $n=8$, then $\omega \approx 1+\sqrt{-1}$. Sinee $N(1+\omega)=\sqrt{2} v$ for some wit $v$ of $D, 1+\omega$ cannot normalize an Ibichler ordor of levol $\delta$. Trenee $\alpha \sim 1+$ $+\sqrt{-1}$ is the only possibility.

If $n=12$, then $\alpha \simeq \zeta \sqrt{-1}$ or $\alpha \simeq 1$ is $\zeta \sqrt{-1}$.

3. In the preceding section wo determined the following list of possible $\alpha$ :

T. $a \approx \sqrt{-m u}$, where $m$ is a scruareftres integen dividing $b / A_{K}$, and $u$ is a totally positive uniti of $\mathfrak{D}$.

II. $\alpha \simeq 1+\sqrt{-1}$ provided $2 \mid \delta A_{K}$.

III. $\alpha \simeq \zeta ; \alpha \simeq \zeta \sqrt{-3}$ provided $3 \mid \Delta_{K}$.

IV. $a \simeq \zeta \sqrt{-1}$ or $a \simeq 1+\zeta \sqrt{-1}$ provided $D=3$.

V. $\alpha \simeq \eta, \eta^{\prime}$ provided $D=5$.

In this section we determine under what conditions such a sotually occur in the normalizer of an Wichler order of level $\delta$. Our first step is to reduce case $\mathrm{T}$.

Limar 1. If $\| \neq 1$, then $\sqrt{-m u} \simeq \sqrt{-m(t a n-2)}$.

Prool. If $\alpha^{2}=-m u$, put $\beta=\alpha(\bar{u}-1)$. Then $\beta^{2}=-m($ tr $u-2)$.

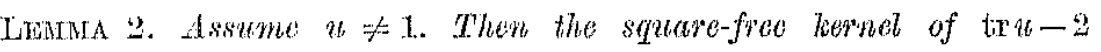
aivides $A_{k}$.

Pro of. Put $u=a+b \sqrt{D}$, where $a, b \in \frac{1}{2} Z, b \neq 0$. Since $a^{2}-b^{2} D=1$. we have

$$
\left(a^{2}-1 . \cdots(a-1)(a+1)=\cdots b^{2} D .\right.
$$

If $D=2,3(\bmod 4)$, then $a, b \in Z$ and $(a-1, a+1)=1$, an acording as $a$ is even or odit, resp. If. $a$ is odd, then (3) implies $b$ is even and

$$
((a-1) / 2)((a+1) / 2)=(b / 2)^{2} D .
$$

Hence $a-1=a^{2} d$ or $(a-1) / 2=a^{2} d$ for some $a \in \mathscr{Z}, d \mid D$. Then tru- $u=2(a-1)=a^{2}(2 d)$, or $\operatorname{tr} u-2=\left(4 c^{2}\right) d$, for some $d \mid D$.

If $D$ : $=1$. (nod 4) and $a, b \in Z$, then taking equation (3) mod 4 , we see that $a$ camot be even. Fitnee $(a-1, a+1)=2$ and $\operatorname{tr} u-2=\left(4 c^{2}\right) d$,

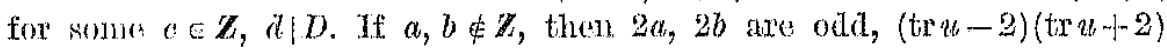
$=(2 b)^{2} D$, and $(\operatorname{tr} u-2, \operatorname{tr} u+2)=1$. It follows that tir $u-2=\sigma^{2} d$, for some $c \in Z, \not d \mid D$.

As a consegnence of the preceding two lemmas, wo have $\sqrt{-m u}$ $\simeq \sqrt{-m^{\prime}}$ for some squarefree divisor $m^{\prime}$ of $\delta \Delta_{K}$. Thus case I reduces to:

I. $a \simeq \sqrt{-m}$, where $m$ is as square-fre integer dividing $\delta \Delta_{K K}$.

We note that, in onch of the cases I-IV, $K(a)$ is a biquadratic extension of $\boldsymbol{Q}$. The matter of whether one of these a ocen's in $\mathfrak{V}_{K}$ is then easily settled by means of the Kronecker symbol. Suppose $K(a)$ $=K(\sqrt{-m})$. Let $A(-m)$ denote the diseriminant of $Q(\sqrt{-m})$. Then

(4) $\quad X^{2}+\dot{m}=0$ solvable in $\mathfrak{R}_{K} \Leftrightarrow\left(\frac{\Delta(-m)}{p}\right) \neq 1$ for all $p \mid \delta_{2}$.

I.1. particular, when $f: 0, x^{*}+m \ldots 0$ is always solvable in $\mathfrak{x}_{K}$.

Now stupose a cosecturs in $\mathfrak{2}_{K}$ and bolongs to one of the cases I-IV. Then, by the eriterion of Eichler ([2], p. 133), the only way a might not oceur in the normalizer of some Eichler order of level $\delta$ is if one of the $p_{i}, i=1, \ldots, e$, remains inest from $K K$ to $K(a)$. Since $K(a)$ is biquadratic over $Q$, this is inmossible. We conclude that a will occur in some normalizer provided only that it occurs a.Jeady in $\mathfrak{A}_{K}$.

As for case $\mathrm{V}$, one readily sees that $\eta$ occurs in $\mathfrak{A}_{K} \Leftrightarrow$ no $q_{j}$ splits completely in $Q(\eta), j=1, \ldots, f \Leftrightarrow q_{j} \not \equiv 1(\bmod 5), j=1, \ldots, f$. Arguing the same as on $p .36$ of [4.], we see that $\eta$ occurs in some normalizer $\Leftrightarrow \eta$ occurs in $\mathfrak{A}_{K}$ and $e=0$. 
4. In this section we determine the contribution to the trace sum (2) of each $\alpha$ which occurs in the normalizer of some Eichler order of level $\delta$. If $\alpha \simeq 1$, then its contribution is $2 M(\mathfrak{I})$, where $M(\mathfrak{I})$ is the weight of $\mathfrak{\Im}$, an explicit formula for which cur be found on $p .26$ of [1].

Now suppose $\alpha \neq 1$, so that $K_{u}=K(\alpha)$ is an imagrinary quadratio extension of $K$. We say that an order $\mathcal{O}$ of $K_{a}$ is admissible for $a$ if $a \in 0$ and, for some twichler order $\Omega$ of level $\delta, \mathscr{O}=\Omega \cap K_{a}$ and $a \in \mathfrak{R}(\Omega)$. We let $l_{0}$ denote the number of primes of $K$ which divide $\delta$ and ramify in $K_{a}$. If $l_{1}$ is the number of $p_{i}, i=1, \ldots$, end $l_{2}$ is the number of $q_{j}$, $j=1, \ldots, f$, which ramify in $K_{\alpha}$, then it is clear that

$$
l_{a}=l_{1}+2 \cdot 2 l_{2} \text {. }
$$

Proceeding exactly as in $\$ 8$ of [4], we find thut the contribution of $a$ to the trace sum is

$$
\begin{aligned}
& \frac{2^{f-1-l_{a} h\left(K_{a}\right)}}{h(K)\left[E_{a}: E_{K}\right]} \sum_{\mathcal{C}}\left[U_{a}^{1}: U^{1}(\tilde{O})\right] \text { if a is puro, }
\end{aligned}
$$

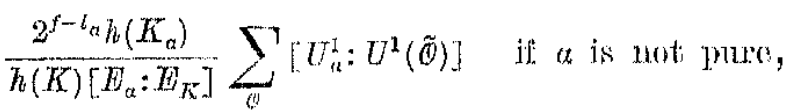

where $O$ ranges over all orders of $H_{\alpha}$ whiteld are admissible for a, and the rest of the notation is as in $\S 8$ of [4] (Noto: $l_{12}=l_{0}$ of $\$ 8$ as tho conduotor condition in the definition of $l_{0}$ is supertluous).

If $n$ is a positive integer, let $h(-n)$ denote the olass mumber of $Q(\sqrt{-n})$ Let $W_{\alpha}$ denote the grom of roots of unity contained in $K_{\alpha}$. If a belongs to one of the case I-IV, then $K_{\alpha}=K(\sqrt{-m})$ for some $m \mid \delta A_{K \text { K }}$, and Bachmann's formula for the class number of an imaginary bicuadratic number field shows that the contribution of a is

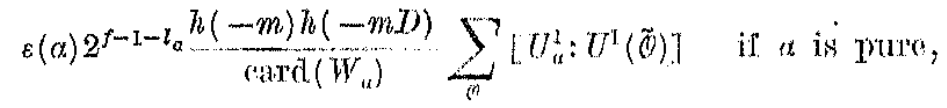

$$
\begin{aligned}
& \text { (8) } \varepsilon(\alpha) 2^{f-l_{\alpha}} \frac{h(-m) h(-m I))}{\operatorname{card}\left(W_{a}\right)} \sum_{a}^{T}\left[f_{a}^{1}: U^{1}(\tilde{a})\right] \text { if } u \text { is noti pure, }
\end{aligned}
$$

where $s(a)=2$ if $K_{a}=Q(\sqrt{-1}, \sqrt{-3})$ and $s(a) \cdots 1$ otherwise, ancl $a$ ranges over all orders admissible for $\alpha_{\text {. }}$

In order to evaluato the entributions (7), (8) explisitiy, one proceeds as in $\$ 8$ of [4]. The firsit step is to determine all the admissible orders for a given $\alpha$. This is done by first cormputing $n_{x / Q}\left(\Lambda_{L / K}\right)$ ([4], Prop. 13, p. 37), where $L=K_{0}$, using this to detormine $A_{\text {man }}$, and then comparing $\Delta_{L / K}$ with $\Delta(-m)$. In the present situation somo eantion must be exer- cised, as $n_{K / Q}\left(\Delta_{L / K}\right)$ ean be divisiblo by $q_{j}^{2}$ for some $j$, and $\Delta_{L / K}$ could a priori be divisible by any product of the two primes of $K$ lying above $a_{j}$. However, in all our cases $\Delta_{L I K}$ is invariant under the conjugation of $K$, which implies $\Delta_{X / K}$ is divisible by $q_{j}$ if its norm is divisible by $q_{j}^{2}$. After all the admissible orders for a given a are determined, the unit indices $\left[U_{a}^{1}: U^{1}(\tilde{\mathcal{O}})\right]$ are computed by means of Proposition 16 of $[4], 1$, 43. The final results are summarized in the formulas of the next section. We omit the details of the computations, as they do not basically differ from the ones carried out in $\$ 8$ of [4].

If ase $V$ occurs, thon $D=5$ and $\theta:=0$. The prinitive fifth roots $\eta, \eta^{\prime}$ each contribute $2^{f} / 5$, so the term $2^{f+1} / 5$ must be added to the trace sum in this case.

5. Ist $m$ be a positive integer. Let $\lambda(m)$ denote the number of primes of $K$ which divide $m$. Dofine $\varepsilon_{m}$ to be 1 if $X^{2}+m=0$ is solvable in $\mathfrak{A}_{E}$ and 0 otherwise.

THEoRman. Let $\mathfrak{I}$ be an idealcomplen of definite maximal Z-lattices of rank four. Let $H$ bo the number of proper similitude elasses in $\Im$. Assume that the reduced discriminant 4 of $\mathfrak{I}$ is not a perfect square and put $K=Q(\sqrt{1})$, so that $A=\Lambda_{K} \delta^{2}$ with $\delta$ square-free. Write $\delta=\delta_{1} \delta_{2}$, where $\delta_{1}$ is only divisible by primes which are inert in $K$, and $\delta_{2}$ is only divisible by primes which split in $K$. Let $f$ be the number of rational primes which divide $\delta_{2}$. Denote the square-free ternel of $\Delta$ by $D$.

(a) If $D=1$ (nod 8), then

(9) $\quad H=2 M(\Im)+$

$$
+2^{f}\left(o_{3} h(-3 D)+\sum_{n|\delta, d| D} \varepsilon_{n d} 2^{-\lambda(n)-a(n d)} h(-n d) h(-n D / d)\right),
$$

where nd $\neq 1,3 ; a<\sqrt{D}$

$$
c_{3}=\left\{\begin{array}{lll}
\frac{1}{6} s_{3} & \text { if } & 3+\Delta, \\
\frac{5}{6} c_{3} & \text { if } & 3 \mid \delta_{1}, \\
5 & \text { if } & 3 \mid \delta_{2}, \\
\frac{5}{12} c_{3} & \text { if } & D \cdot 3=3(\bmod \theta), \\
\frac{7}{3} \varepsilon_{3} & \text { if } & D=6(\bmod 9), \\
2 c_{3} & \text { if }
\end{array}\right.
$$

and

$$
\sigma(m)=\left\{\begin{array}{lll}
-2 & \text { if } & m=3(\bmod 8), \\
0 & \text { if } & m=7(\bmod 8), \\
2 & \text { if } & m=2(\bmod 4), \\
2 & \text { if } & m \equiv 1(\bmod 4), 2+\delta_{z}, \\
4 & \text { if } & m \equiv 1(\bmod 4), 2 \mid \delta_{2} .
\end{array}\right.
$$


(b) If $D=5(\bmod 8), D \neq 5$, then

$$
\begin{aligned}
& H=2 M(\Im)+2 f\left(a_{1} l_{b}(-D)+c_{3} b_{b}(-3 I)\right)+
\end{aligned}
$$

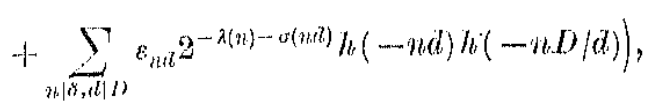

where $u d \neq 1,3 ; d<\sqrt{D}$

$$
\begin{gathered}
c_{1}= \begin{cases}\frac{1}{8} \varepsilon_{1} & \text { if } \quad 2 \nmid \delta_{1}, \\
\frac{3}{16} \varepsilon_{1} & \text { if } \quad 2 \mid \delta_{1},\end{cases} \\
c_{3}=\left\{\begin{array}{lll}
\frac{1}{6} \varepsilon_{3} & \text { if } & 3 \nmid A, \\
\frac{1}{3} \varepsilon_{3} & \text { if } & 3 \mid \delta_{1}, \\
\frac{1}{6} \varepsilon_{3} & \text { if } & 3 \mid \delta_{2}, \\
\frac{4}{3} \varepsilon_{3} & \text { if } & D=3(\bmod 9), \\
\varepsilon_{3} & \text { if } & J)=6(\text { mod } 9),
\end{array}\right.
\end{gathered}
$$

$\operatorname{and}$

$$
\sigma(m)==\left\{\begin{array}{lll}
0 & \text { if } & m=3(\bmod 4), \\
2 & \text { if } & m=2(\bmod 4), \\
2 & \text { if } & m=1(\bmod 4), 2+\delta_{1}, \\
3 & \text { if } & m=1(\bmod 4), 2 \mid \delta_{1} .
\end{array}\right.
$$

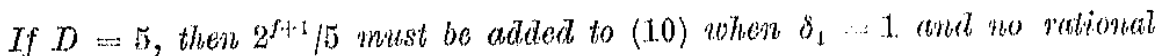
prime dividing $\delta_{2}$ is $\equiv 1$ (mod 5$)$.

(c) If $D \equiv 3(\bmod 4)$, then for avery $n|\delta, d| . D$, enther nal or $n D / d$ is $: 3(\bmod 4)$; if $D \neq 3$, then

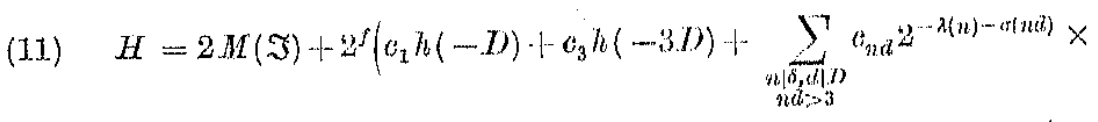

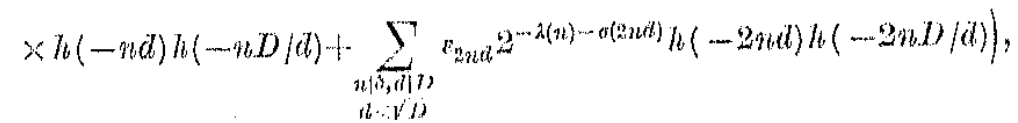

where

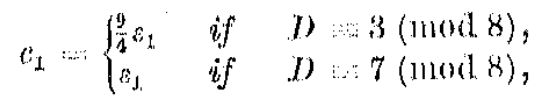

$$
\begin{aligned}
& \theta_{3}=\left\{\begin{array}{lll}
\frac{1}{6} \varepsilon_{3} & \text { if } & 3 \uparrow A, \\
\frac{7}{12} \varepsilon_{3} & \text { if } & 3 \mid \delta_{1}, \\
\frac{7}{21} \varepsilon_{3} & \text { if } & 3 \mid \delta_{2}, \\
\frac{11}{6} \varepsilon_{3} & \text { if } & D=3(\bmod 9), \\
\frac{3}{2} \varepsilon_{3} & \text { if } & D=6(\bmod 9),
\end{array}\right.
\end{aligned}
$$

and

for $m>3$,

$$
c_{m}- \begin{cases}0 & \text { if } \quad m \neq 3(\bmod 4) \\ 5 \varepsilon_{m} & \text { if } \quad m=3(\bmod 8) \\ \varepsilon_{m} & \text { if } \quad m=7(\bmod 8)\end{cases}
$$

$$
\sigma(m)=\left\{\begin{array}{lll}
0 & \text { if } & m=7(\bmod 8), \\
1 & \text { if } & m=3(\bmod 8), \\
2 & \text { if } & m=2(\bmod 4) .
\end{array}\right.
$$

If $D=3$, then $\varepsilon_{1}=\varepsilon_{3}$, and the torm $2^{f}\left(c_{1} h(-D)+c_{3} h(-3 D)\right)$ in (11) must be replaced by $2^{f}\left(\frac{17}{12} \varepsilon_{1}\right)$.

(d) If $D \equiv 2(\bmod 4)$, then

(12) $\quad H=2 M I(\Im)+2 f\left({ }_{8}^{5} \varepsilon_{1} h(-D)+c_{3} h(-3 D)+\right.$

$$
\left.+\sum_{\substack{n|s, d| D \\ l d o d d}} e_{n d^{2}} 2^{-\lambda(n)-q(n(n))} h(-n d) h(-n D / d)\right),
$$

where $n d>3, c_{3}$ is as in part (c), and for $m>3$,

$$
\begin{gathered}
c_{m}=\left\{\begin{array}{lll}
5 \varepsilon_{m} & \text { if } & m \equiv 3(\bmod 8), \\
\varepsilon_{m} & \text { if } & m \equiv 7(\bmod 8), \\
3 \varepsilon_{m i} & \text { if } & m \equiv 1(\bmod 4),
\end{array}\right. \\
\sigma(m)=\left\{\begin{array}{lll}
1 & \text { if } & m \equiv 3(\bmod 8), \\
0 & \text { if } & m \equiv 7(\bmod 8), \\
2 & \text { if } & m \equiv 1(\bmod 4),
\end{array}\right.
\end{gathered}
$$

Concluding remarks. The above formulas can be interpreted in the classical language of quadratic forms (cf. [4], p. 32-33). In particular, if $\delta=1$, then $H=$ the number of proper classes of positive definite integral quaternary forms with discriminant $\Delta_{T}$.

In [5] Vignéras obtained formulas for the arithmetic genus of certain Frilbert modular varieties, and claimed (Theorème, p. 212) that, as a special case, one obtained a formula for the trpe number $t$ in the case $\delta=1$. Our resultis for the case $\delta=1$ show that this formula is not, in general, a formula for $t$, as it does not contain the contributions of all the elements $\alpha \simeq \sqrt{ }=a$, where $a \mid \Delta_{K}$. The source of the difficulty is that the ideal class number of $\mathfrak{A}_{K}$ divided by the proper class number of $K$ is not the type number $t$, in general, ovon if $\mathfrak{A}_{K}$ is split at all finite primes of $K$.

\section{References}

[1] H. Cohn, A second course in number theory, Wiley, New York-London 1962

[2] M. Eichlor, Zur Zahlentheorie iler Quaternionen-Algebren, J. Reine Angew. Math. 195 (1955), pp. 127-151. 
[3] P. Ponomarev, Class numbers of definite quaternary forms with square diso minant, J. Number Theory o (1974), pp. 291-317.

[4] - Arithmetio of quaternary quadratio forms, Acta. Arith. 29 (1976), pp. I-

[5] M. F. Vignóras, Invariants mumériques des groupes de Hillert, Matle. A 224 (1976), pp. 189-21\%

\section{DEPATTMLENT' OF MATHIBMATIO}

TTER OFIO STATIL UNEYHRSITY

Columbus, olio 43210

Received on 26.8. 1978

and in revised form on 30.70.7978

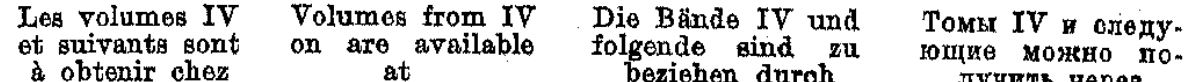

\section{Ars Polona, Krakowskie Przedmiescie 7, 00-068 Warazawa} Les volumes I-III Volumes I-III
sont a obtenir chez are available at
zu beziehen durch Johnson Reprint Corporation, 111 Fifth Ave., Now Yoxk, N. X.

\section{BOOKS PUBLISHED BY THE POLIST AOADEMTY OF SOTENOES} INSTITUTE OF MATHEMCATIOS

S. Banach, Oetrvres, vol. II, $1979,470 \mathrm{pp}$.

S. Mazurkiewioz, Travaux do topologio et ses applications, 1968, $380 \mathrm{pp}$.

W. Sierpiriski, Oeuvres ohoisies, vol. I, 1974, 300 pp.; vol. II, 1875, 780 pp.; rol. III, 1976, $688 \mathrm{pp}$.

J. P. Schauder, Oeurres, 1978, 487 pp.

H. Steinhaus, Selected papers, in print.

Proceedings of the Symposium to honour Jerzy Neyman, 1977, $349 \mathrm{pp}$.

Proceodings of the International Conference on Geometric Topology, 1980, $467 \mathrm{pp}$

\section{MONOGRAFIE MATEMATYCZNE}

27. K. Kuxatowski i A. Mostowski, Teoria mnogosei, 5th ed., 1978, 470 pp.

43. J. Szarski, Differential inequalities, 2nd ed., 1967, $256 \mathrm{pp}$.

44. K. Borsuk, Theory of retracts, 1967, $251 \mathrm{pp.}$

45. K. Maurin, Methods of Hilbert spaces, 2nd ed., 1972, $552 \mathrm{pp}$.

47. D. Przeworska-Rolewioz and S. Rolewioz, Equations in linear spaces, $1968,380 \mathrm{pp}$.

50. K. Borsu.k, Multidimensional analytic geometry, $1969,443 \mathrm{pp}$.

51. R. Sikorski, Advanced caloulus. Functions of several variables, $1969,460 \mathrm{pp}$.

58. C. Bessag a and A. Pelozyriski, Selected topics in infinite-dimensional topology, $1975,353 \mathrm{pp}$.

59. K. Borsuk, Theory of shape, $1975,379 \mathrm{pp}$

60. R. Engelking, General topology, 1977, 626 pp.

61. J. Dugundji and A. Granas, Fixed point theory, vol. I, in print

\section{BANACH CENTER PUBLTCATIONS}

Vol. 1. Mathematioal control theory, 1976, $166 \mathrm{pp}$.

Vol. 4. Approximation theory, 1978, $314 \mathrm{pp}$.

Vol. 5. Probability theory, 1979, $289 \mathrm{pp}$

Vol. 6. Mathematical statistios, 1980, $376 \mathrm{pp}$.

Vol. 7. Discrete mathematics, in print.

Vol. 8. Spectral theory, in print.

Vol. 9. Universal algebra and applications, in print.

Vol. 10. Partial differential equations, in print.

Vol, 11. Complex analysis, in print. 